\title{
The Global Spine Care Initiative: model of care and implementation
}

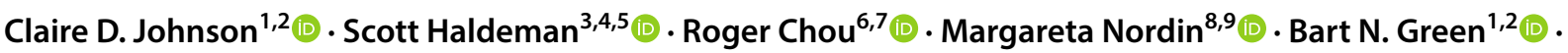 \\ Pierre Côté ${ }^{10,11}$ - Eric L. Hurwitz ${ }^{12}$ (D) Deborah Kopansky-Giles ${ }^{13,14}$ (Emre Acaroğlu ${ }^{15}$ (1) . \\ Christine Cedraschi ${ }^{16,17}$ (1) Arthur Ameis ${ }^{18}$ (1) $\cdot$ Kristi Randhawa $^{10,11}$ (1) Ellen Aartun ${ }^{11} \cdot$ Afua Adjei-Kwayisi $^{19}$ (1) \\ Selim Ayhan ${ }^{20}$ - Amer Aziz ${ }^{21} \cdot$ Teresa Bas $^{22}$. Fiona Blyth ${ }^{23} \cdot$ David Borenstein $^{24}{ }^{(1)} \cdot \mathrm{O}^{\prime}$ Dane Brady ${ }^{25}$ (D) \\ Peter Brooks ${ }^{26}$. Connie Camilleri ${ }^{27} \cdot$ Juan M. Castellote ${ }^{28} \cdot$ Michael B. Clay $^{29} \cdot$ Fereydoun Davatchi $^{30}$. \\ Jean Dudler ${ }^{31}$ (D) Robert Dunn ${ }^{32}$ (1) . Stefan Eberspaecher ${ }^{33}$. Juan Emmerich ${ }^{34}$. Jean Pierre Farcy ${ }^{35}$. \\ Norman Fisher-Jeffes ${ }^{36}$. Christine Goertz ${ }^{37}$ (1) Michael Grevitt ${ }^{38} \cdot$ Erin A. Griffith $^{39} \cdot$ Najia Hajjaj-Hassouni $^{40}{ }^{(1)}$. \\ Jan Hartvigsen ${ }^{41,42}$ (D) Maria Hondras $\mathrm{s}^{43}$ (1) . Edward J. Kane $\mathrm{K}^{44}$ (D) . Julie Laplante ${ }^{45}$. Nadège Lemeunier ${ }^{46}$. \\ John Mayer $^{47} \cdot$ Silvano Mior ${ }^{48} \cdot$ Tiro Mmopelwa $^{49} \cdot$ Michael Modic $^{50} \cdot$ Jean Moss $^{48} \cdot$ Rajani Mullerpatan $^{51}$. \\ Elijah Muteti $^{52} \cdot$ Lillian Mwaniki $^{53} \cdot$ Madeleine Ngandeu-Singwe $^{54} \cdot$ Geoff Outerbridge $^{55}$ (1). \\ Shanmuganathan Rajasekaran ${ }^{56} \cdot$ Heather Shearer $^{11} \cdot$ Matthew Smuck $^{57} \cdot$ Erkin Sönmez $^{58}$ (D) Patricia Tavares ${ }^{48}$. \\ Anne Taylor-Vaisey ${ }^{11}$. Carlos Torres ${ }^{59} \cdot$ Paola Torres $^{60}$. Alexander van der Horst ${ }^{61,62} \cdot$ Leslie Verville $^{10,11}$. \\ Emiliano Vialle ${ }^{63}$. Gomatam Vijay Kumar ${ }^{64}$. Adriaan Vlok ${ }^{65} \cdot$ William Watters III $^{66,67}$. Chung Chek Wong ${ }^{68}$. \\ Jessica J. Wong ${ }^{11} \cdot$ Hainan $\mathrm{Yu}^{10,11}$ (1) Selcen Yüksel ${ }^{69}$
}

Received: 12 April 2018 / Accepted: 6 August 2018 / Published online: 27 August 2018

(c) The Author(s) 2018

\begin{abstract}
Purpose Spine-related disorders are a leading cause of global disability and are a burden on society and to public health. Currently, there is no comprehensive, evidence-based model of care for spine-related disorders, which includes back and neck pain, deformity, spine injury, neurological conditions, spinal diseases, and pathology, that could be applied in global health care settings. The purposes of this paper are to propose: (1) principles to transform the delivery of spine care; (2) an evidence-based model that could be applied globally; and (3) implementation suggestions.

Methods The Global Spine Care Initiative (GSCI) meetings and literature reviews were synthesized into a seed document and distributed to spine care experts. After three rounds of a modified Delphi process, all participants reached consensus on the final model of care and implementation steps.

Results Sixty-six experts representing 24 countries participated. The GSCI model of care has eight core principles: personcentered, people-centered, biopsychosocial, proactive, evidence-based, integrative, collaborative, and self-sustaining. The model of care includes a classification system and care pathway, levels of care, and a focus on the patient's journey. The six steps for implementation are initiation and preparation; assessment of the current situation; planning and designing solutions; implementation; assessment and evaluation of program; and sustain program and scale up.

Conclusion The GSCI proposes an evidence-based, practical, sustainable, and scalable model of care representing eight core principles with a six-step implementation plan. The aim of this model is to help transform spine care globally, especially in low- and middle-income countries and underserved communities.
\end{abstract}

Electronic supplementary material The online version of this article (https://doi.org/10.1007/s00586-018-5720-z) contains supplementary material, which is available to authorized users.

Claire D. Johnson

globalspinecareinitiative@gmail.com

Extended author information available on the last page of the article 
Graphical abstract These slides can be retrieved under Electronic Supplementary Material.
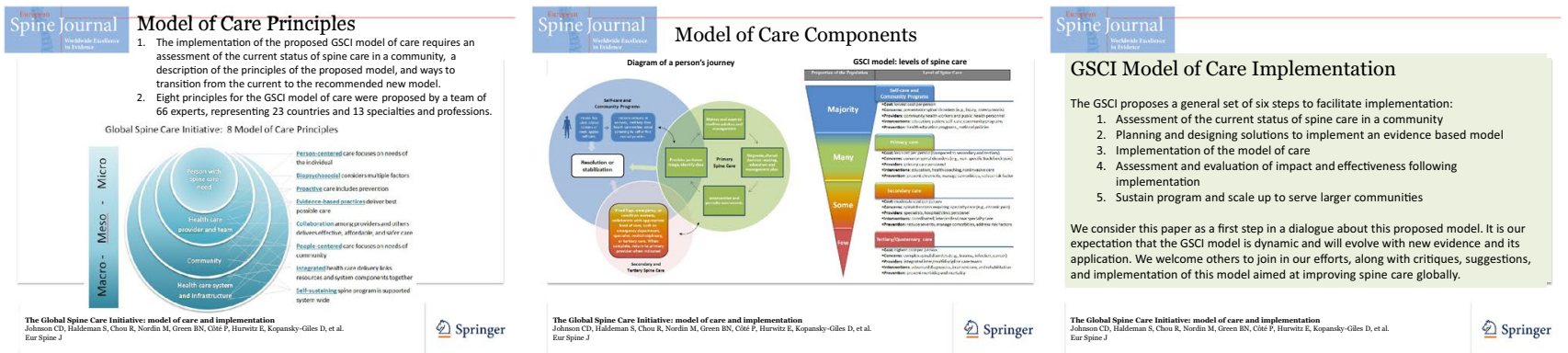

Keywords Global burden of disease · Musculoskeletal system · Spinal diseases · Quality of health care

\section{Introduction}

Spinal disorders are a leading source of global disability and result in a considerable burden on society [1-4]. People in low- and middle-income countries and those in communities under socioeconomic stress are especially affected $[5,6]$. Globally, years lived with disability caused merely by low back pain increased by $54 \%$ between 1990 and 2015, mainly because of population increase and aging, with the biggest increase seen in low-income and middle-income countries [7]. In some countries, back pain is estimated to contribute to up to $30 \%$ of direct costs (e.g., costs associated with health care) and indirect costs (economic consequences of the illness such as loss of work and productivity) [8] are estimated to represent the majority of overall costs [9]. Costs of care for back pain vary among countries depending on the type of study; however, total costs demonstrate a substantial financial burden regardless of the variation [8]. Similar problems exist for other spinal disorders.

While searching for solutions to these issues, it must be kept in mind that the world faces a shortage of health resources and professionals to address the increasing burden of chronic and non-communicable diseases. These issues may be especially troublesome in underserved areas and low- and middle-income countries [1, 10-14]. In these regions, there is a risk of overuse or inappropriate use of urgent or specialty care to manage common spinal conditions such as non-specific back pain. This reduces available resources that should more appropriately be used for other health care needs. The concern is that inappropriate use of health care drains resources away from those who may need it most [15]. As well, there is concern that if approaches used in some high-income countries are applied to other world regions, it could create demands for costly or ineffective health care procedures that increase the risk of longterm disability [16]. These issues need to be considered when developing solutions.
Despite these facts, spinal health care has not received the attention that is needed to make a change. However, a shift in the health care paradigm may help transform how we address chronic and non-communicable conditions, as they are finally being recognized as a global burden and priority [17-22]. For example, a call for action has demanded changes in policy, public health, health care practices, social services, and workplaces to tackle the low back pain paradox in low-income and middle-income countries [23].

Thus, there are important health care needs for individuals and compelling concerns of public welfare, community economics, and social justice. Unfortunately, there is no comprehensive, evidence-based model of care that considers these critical issues when providing guidance to address spinal disorders. A pragmatic model of spine care is needed to accommodate conditions that are seen by a variety of health practitioners in a variety of settings. Since people have spine-related concerns ranging from the most benign to potentially life-threatening, a broad model is essential to address all possible spinal disorders, not only one type of spine-related concern (i.e., back pain) or condition (i.e., osteoporosis). Because spine-related concerns are multifactorial, a model is needed that can address issues for the individual/patient and the community; thus, it should contain preventive and self-care programs that are integrated into a comprehensive model of care to manage spinal disorders.

While the need for such a model is great and pressing, pragmatic implementation steps are required so that planners may adapt the proposed model to fit available local resources in any world region. We are unaware of any such implementation plan. For communities with few resources, deployment of a plan should be flexible. An ideal plan would also allow for expansion as spine care needs are identified and as more resources become available. Local implementation should aim to improve spine care delivery, reduce costs per individual, and reduce the burden of spinal disorders based upon community needs. 
This paper attempts to fill these critical breaches in global spinal health care delivery and research. The purposes of this paper are to propose: (1) principles to transform the delivery of spine care; (2) an evidence-based model that could be applied globally; and (3) implementation suggestions.

\section{Methods}

The GSCI team was assembled as described in the methodology paper [24]. In summary, a series of meetings were convened to frame the scope of the project Chicago, Illinois (July 2014) Toronto, Canada (November 2014), and San Jose, California (March 2015). During these meetings, scope, topics, and goals for the papers were finalized. After the first set of review papers were completed, we drafted the evidence-based model of care based through a consensus processes. The National University of Health Sciences (Lombard, IL, USA) institutional review board reviewed the modified Delphi process and approved this process (\#H1503). All panel experts who responded provided consent to participate.

A modified Delphi process was utilized to obtain consensus from the Global Spine Care Initiative (GSCI) experts [25-27]. The working groups were comprised the 68 authors from the GSCI papers [1-3, 24, 28-37], representing experts from 24 countries. Fifty-seven of these authorities possessed experience in providing spine care in 34 countries (i.e., high-, middle-, and low-income countries, as well as underserved communities in high-income countries [24]). The experts represented the fields of specialty medicine (e.g., neurology, orthopedic spine surgery), generalist medicine, physiotherapy, nursing, chiropractic, epidemiology, statistics, higher education, library sciences, law, and anthropology.

A seed document was crafted by synthesizing information from notes taken by the lead author (CJ) at the GSCI meetings [24] and the GSCI papers [1-3, 28-31, 38]. The seed document included core principles, a model of care and recommendations for implementation steps. The document was distributed to the expert panel by the electronic survey platform Survey Monkey (SurveyMonkey Inc., San Mateo, California, USA). The first round collected responses to open-ended questions. The response materials were gathered and evaluated to identify areas of disagreement and consensus. The document was revised, and the revision was redistributed to the group for further review and comment. Because the second round had a majority of agreement and little substantive feedback, the third round of review asked, "If you agree overall with the contents of the paper (whether or not you have additional feedback), please type I agree". In the third round, no further substantive feedback was offered.

\section{Results}

Sixty-six of the 68 GSCI team members participated and contributed to this document, which is a $97 \%$ response rate. The experts represent 13 specialties/professions. The following is a description of the GSCI model of care and suggestions for implementation. All participating experts agreed on the final report represented in this paper, which we designated to be a consensus.

\section{Model of care principles}

A model of care helps to transform a health care system to address a given health concern [39]. Davidson defines a model of care as

$\ldots$ an overarching design for the provision of a particular type of health care service that is shaped by a theoretical basis, EBP and defined standards. It consists of defined core elements and principles and has a framework that provides the structure for the implementation and subsequent evaluation of care. Having a clearly defined and articulated model of care will help to ensure that all health professionals are all actually 'viewing the same picture', working toward a common set of goals and, most importantly, are able to evaluate performance on an agreed basis. [40]

For a transformation process to succeed, underlying principles should be clearly defined to direct the transition from the current model of care into the new model of care. The following are eight principles that the GSCI expert panel agreed would facilitate the transition from an old to the new GSCI model of care (see Fig. 1).

\section{Transform from provider-centered to person-centered}

In a "provider-centered" model, the patient lacks autonomy and relies on the provider for decisions and care. The perception is that without the health care provider no healing or health could be achieved. Success metrics focus on the providers (e.g., number of procedures delivered) or may be the number of people that a provider sees with a certain condition (e.g., number of osteoporosis cases). The focus is on the provider's thoughts (e.g., diagnosis, management decisions) and actions (e.g., surgery, drugs, manual/physical therapies, and passive therapies) and patients are seen as "cases" or "conditions" not as whole beings.

The GSCI model of care values person-centered care. Person-centered care empowers the person (or patient) to participate in the healing process [41-43]. The provider respects the individual's autonomy and includes the individual's culture, beliefs, and values in spinal health care decisions [29]. 
Person-centered care provides individuals with information to help them decide when to seek care, when to engage in self-care, and increases the likelihood of patient participation and compliance with the treatment plan, resulting in better outcomes, and costs. Person-centered care includes effective communication, shared decision making, holistic care, individualized care, and continuity of care [44, 45]. Person-centered success metrics focus on the spinal health of individuals and provide information about if the patients are receiving the correct care and if their health is improving. Ongoing assessment and monitoring of person-centered care delivery and systems offers objective results and encourages improvement [46, 47]. Evaluation increases awareness of processes and actions that are in alignment with best practices and could promote improved care and research [48].

\section{Transform from system-centered to people-centered}

System-centered health care focuses on business aspects or the organization that administers or houses the care. Metrics might include the number of people treated or hospital night stays, instead of serving the needs of the community. Such a focus on the delivery of care, instead of on the end-users, typically does not include community stakeholders in decisions.

The GSCI model emphasizes people-centered health care systems, which refocuses the health care system on the needs of the people in the community and society. In this model, the system engages community representatives as stakeholders and part of the health care relationship in the health care system
[49]. People-centered, culturally competent health care can be integrated throughout the system such as through educational interventions, clinical encounters, health promotion programs and services, and processes at the systems level [50]. Peoplecentered care contains a wide variety of community participants including the individual, care givers, families, and communities as participants in the health care process and includes not only the clinical encounter but also health policy and services [51]. People-centered care is participatory; therefore, there is greater accountability to local stakeholders and disadvantaged populations [46]. Success metrics include those related to community health, such as population health outcome measures and value of care indicators.

\section{Transform from biomedical to biopsychosocial}

The biomedical model has over-emphasized spinal pathology as a cause for spine pain (e.g., disk herniation, joint arthritis, and muscle strain), which has led to an overemphasis on pain management. A focus on pain leads to treatments to address pain, which tend to be passive (e.g., analgesics, massage, surgery) instead of active (e.g., therapeutic exercise, active care plans). Passive methods run the risk of addictive side effects (i.e., opioids), potential adverse reactions (i.e., non-steroidal anti-inflammatory drugs cause gastric bleeding), or dependency upon the provider for pain control (i.e., excessive or long-term use of massage and manual therapy) instead of the person being a more active participant in maintaining his/her health [52]. Back pain treated

\title{
Global Spine Care Initiative: 8 Model of Care Principles
}
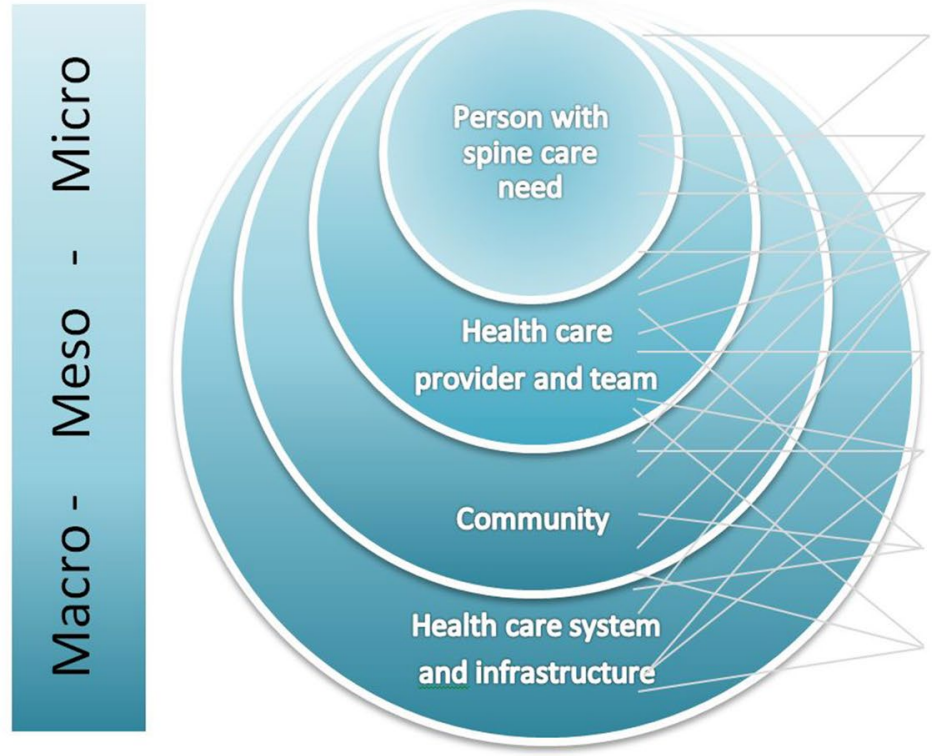

\author{
Person-centered care focuses on needs of \\ the individual \\ Biopsychosocial considers multiple factors \\ Proactive care includes prevention \\ Evidence-based practices deliver best \\ possible care \\ Collaboration among providers and others \\ delivers effective, affordable, and safer care \\ People-centered care focuses on needs of \\ community \\ Integrated health care delivery links \\ resources and system components together \\ Self-sustaining spine program is supported \\ system wide
}

Fig. 1 GSCI principles. Reproduced with permission by World Spine Care 
as a disease, even when there is no identifiable pathological cause of the pain, can lead to poor psychosocial outcomes and inappropriate use of treatments including analgesic medications, such as opioids and more invasive treatments such as injections or surgery and may also facilitate acute conditions to become chronic. This model also risks turning people with no severe pathology into "patients" and thereby increasing costs of spine care with little impact on the community burden of disease. The traditional biomedical model has included little consideration for local population needs, culture, or psychosocial factors that may be relevant to the healing processor prevention.

In the GSCI model, the biopsychosocial model for spine care considers the complex interaction among biological, psychological, and social factors that influence spinal health, disease, and disability. The biopsychosocial model considers how various determinants of health interact and that spinerelated disorders are complex and multifactorial [53, 54]. Determinants of health help to identify the potential contributors of spinal conditions and functional impairment by considering other risk factors or comorbidities that could contribute to pain, disability, or spinal disease. Addressing biopsychosocial factors empowers individuals and communities to be more engaged in self-awareness, self-care, and know when to seek care and when not to seek care for a spine-related concern. A de-emphasis on pain, recognizing that some back and neck pain may be considered normal, helps individuals refocus on functional ability and their values. An emphasis on outcomes of function helps to bolster empowerment for patients and participants in their spinal health. Comprehensive care approaches the whole patient and coordinates or integrates services of many professionals working together to address biopsychosocial needs.

\section{Transform from reactive to proactive}

Reactive health care is when the health care provider or system waits for a disease or injury to occur before any action takes place, which results in the treatment of mainly acute back and neck pain and spinal injuries. While reactive spine care addresses an immediate concern, there are few efforts toward preventing spinal conditions or injuries from occurring or worsening. Focusing only on immediate concerns is perceived to conserve resources but may run the risk of ignoring the potential for a person's condition to become chronic or for secondary issues to arise (e.g., addiction to pain medications). Reactive health care systems are primarily focused on disease treatment and typically do not include prevention strategies, which may put an additional burden on low-income or vulnerable populations [55].

The GSCI model values proactive spine care, thus in addition to acute care, prevention, early diagnosis, and appropriate self-care and treatment are incorporated throughout health care delivery and public programs [48]. Proactive care integrates primary spine care with public health principles which include the "art and science of promoting and protecting good health, preventing disease, disability and premature death, restoring good health when it is impaired by disease or injury and maximizing the quality of life" [55]. Public health activities are implemented through the collective action of a wide variety of people including patients, health care providers, community health and social workers, administrators, educators, government officials, and many other professionals. The levels of prevention included in the model of care are primary prevention (e.g., community outreach to prevent spine injuries/conditions), secondary prevention (e.g., prevent re-injury, prevent acute spine pain from becoming chronic) tertiary prevention (e.g., prevention from worsening conditions-resulting in cost savings and reduced use of health care resources by avoiding resource demanding care) and quaternary prevention (preventing the medicalization or over-medicalization of health concerns) [32]. It is especially important to incorporate quaternary prevention since it has been shown when Western medicine is introduced, some people may turn into "patients" unnecessarily [56]. Preventive health interventions are proactive and can be delivered at an individual or community level and at various stages of spine care. The WHO global strategy on integrated people-centered health services recommends strategic goals, which includes health promotion, disease prevention, and public health [57]. Preventive care and community health programs aim to reduce the burden of health disorders to society and, as the WHO model suggests, may cost the least on average per person compared to tertiary level care [58].

5. Transform from eminence-based to evidence-based practices

"Eminence-based medicine" describes the practices and procedures that are based on the opinions of the care provider that may or may not be based in best evidence [59], which may result in the use of ineffective, harmful, or unnecessary and costly procedures. Examples in spine care may include excess use of pharmaceuticals for mild back pain, overuse of passive modalities, or inappropriate indications for surgery. This may result in unnecessary procedures, increased risk for harm, decreased health benefits, increased direct and indirect costs to individuals and the community, and increased patient dependence on health care providers.

Evidence-based practices balance and combine the relationships between the person, provider, and evidence. The triad of evidence-based care (i.e., the combination of best evidence, provider expertise, and patient values) helps to ensure the best care for spine-related concerns [46, 60, 61]. It must not be assumed that traditional healing practices that 
prevent or treat spinal conditions are absent or ineffective; thus, thorough research should be done to find out which kinds of spine problems exist, how people are dealing with them, and what seems to be effective. By using evidencebased practices, practitioners stay updated with current practices through feedback and education. Communities and individuals are kept abreast of current evidence-based methods for self-care, information about when to seek care, and knowledge of what to expect from providers once they have entered the health care system.

\section{Transform from silos to collaboration}

In the clinical practice environment, individual health care practitioners often work in seclusion within their disciplinary silos [62]. This culture results in little communication or collaboration between providers or with patients and others in the community. The result in these situations may be uncoordinated or redundant spine care, which in turn may result in over use of diagnostic procedures, over treatment, and wasted resources.

The culture of collaboration occurs when there is teamwork among health care providers across disciplines and with other providers and stakeholders. Practitioners who collaborate with providers from other disciplines have the potential to reduce redundancy of procedures or consultations and provide more effective, affordable, and safer care [63-65]. Stakeholders in these collaborative relationships may also include governmental leaders, opinion leaders, individuals/patients, families, and others [46].

\section{Transform from isolated to integration}

Many of the system processes on the meso- and macro-levels of spine care delivery are isolated. This isolation results in poor communication and inefficient distribution or linking of resources resulting in loss of quality, higher costs, and poorer outcomes.

Integration has the potential to link resources and system components together to provide best possible care for individuals and the community. The WHO defines integrated delivery as "The management and delivery of health services so that clients receive a continuum of preventive and curative services, according to their needs over time and across different levels of the health system" [66]. Strategies of integration may occur in many ways, such as through systems, organizations, professions, horizontal (integrating similar levels of care) and vertical (integrating different levels of care). The principles of integration also embrace patient-centeredness in that spine care is considered through a person's lifespan, including needs at various ages and coordinated within and across the local health system [46, 67-70]. Integrated care improves quality [71] and includes engagement of local stakeholders with the spine care program and collaboration with public health and community services. Although integrated care has the potential to be costly, components of integration may still be implemented in low-resource settings.

\section{Transform from fragile to self-sustaining}

For some low- or middle-income communities, spine care is delivered using a fragile model. Care may be inserted by a foreign entity or funded as a charity service. Spinal health care may be treated as if it were "missionary work." This type of delivery system may be seen as fragile in that it may result in dependency on others for services and reduces the individual and community capacity to take responsibility. This model can be perceived as a foreign entity exerting its culture and devaluing the local culture of healing practices. This may result in a split or sub-culture of healing, one in which foreigners provide care resulting in the traditional methods going underground.

Self-sustaining programs require policy alignment and system-wide stakeholder engagement for successful implementation of a spine care program. Local and regional government leaders, policy makers, and community stakeholders must have a role in the development of a self-sustaining program. Each community that wishes to implement a spine care program has a unique population of people with spinal disorders, unique cultural priorities, and unique pre-existing health care infrastructure. A spine program must be developed to integrate into the currently available health care resources and culture to be sustainable. Effective delivery of health care services requires efficient financing systems and funding to support knowledgeable and skilled personnel and adequate infrastructure [46]. Sustainability requires "planning, managing, and delivering care that is equitable, efficient, effective" [46].

\section{Model of care principles transition tool}

Before implementing the model of care, we propose that the eight GSCI principles be evaluated for the target location. One way to evaluate the principles is by using a web diagram. This tool provides an assessment and an overall visual representation of the current state of spine care and targeted goals for each of the principles. Each principle is rated on a scale by the steering committee. This tool can help the steering committee team leaders communicate their vision to others when implementing the model. Figure 2 is an example of how an assessment chart might look after a local steering committee has rated the current situation (now) and the goals that they have set for themselves for each principle. Each axis represents one principle and is rated with a numeric scale. Examples of actions that could 
be taken to transition from the current (now) environment to the goal environment are presented in the supplemental file (see Online Resource Figure 1). These are the steps that would be selected based on the resources and community needs during implementation planning.

\section{GSCI model of care}

Based upon the eight principles, the GSCI proposes the following three components that contribute to the model of care, which are: (1) The GSCI classification and care pathway; (2) The GSCI levels of care; (3) The GSCI person's journey. Each of these components is described below.

\section{GSCl classification and care pathway}

The GSCI classification and care pathway were developed to assist with decision making at a patient care level but also provide a structure for all providers, health care consumers, and others engaged in the health care system to use to communicate and work together. The GSCI classification and care pathway accommodates any person's concern related to the spine and is consistent with current spine classifications and guidelines. The classification categorizes a person's presentation so that it may be linked directly with the GSCI care pathway [33,34]. The care pathway provides evidencebased, diagnostic procedures, and treatments that the provider may select from $[3,28,29,31]$. The care pathway includes health promotion and prevention strategies, including primary, secondary, tertiary, and quaternary prevention
[32] and psychological or social factors in patient recovery and wellness to address all levels of care needed by the population [30] (Fig. 3a, b). Goals include preventing future conditions, preventing chronicity, prevention of unnecessary medical procedures or medical dependence, and the quickest possible return to function and normal activities. A detailed description of the GSCI classification and care pathway are described in other papers $[33,34]$.

\section{GSCl levels of care}

The GSCI model of care proposes four levels for providing spine care: self-care and community spine programs; primary spine care; secondary spine care; and tertiary/quaternary spine care (Fig. 4). The GSCI levels of care demonstrate the types of spine care, population needs, and levels of resources required to cover the needs of the community. These stepped levels of care match services with individual needs and may be delivered in a variety of health care settings. Care provided by solo or group health care providers may be included in health centers, outpatient clinics, public health departments, and other ambulatory settings. More advanced care may be provided in emergency rooms, outpatient departments, hospices, ambulatory surgery centers. Care may also be provided in non-health care settings, such as at an individual's place of residence, place of employment, or a community. The level of care should match the setting so that the safest and most effective care may be provided. To be efficient, primary spine care settings should be integrated and coordinated with other
Fig. 2 Graphic representation a sample rating of current state and goals. Each axis represents one of the eight GSCI models of care principles

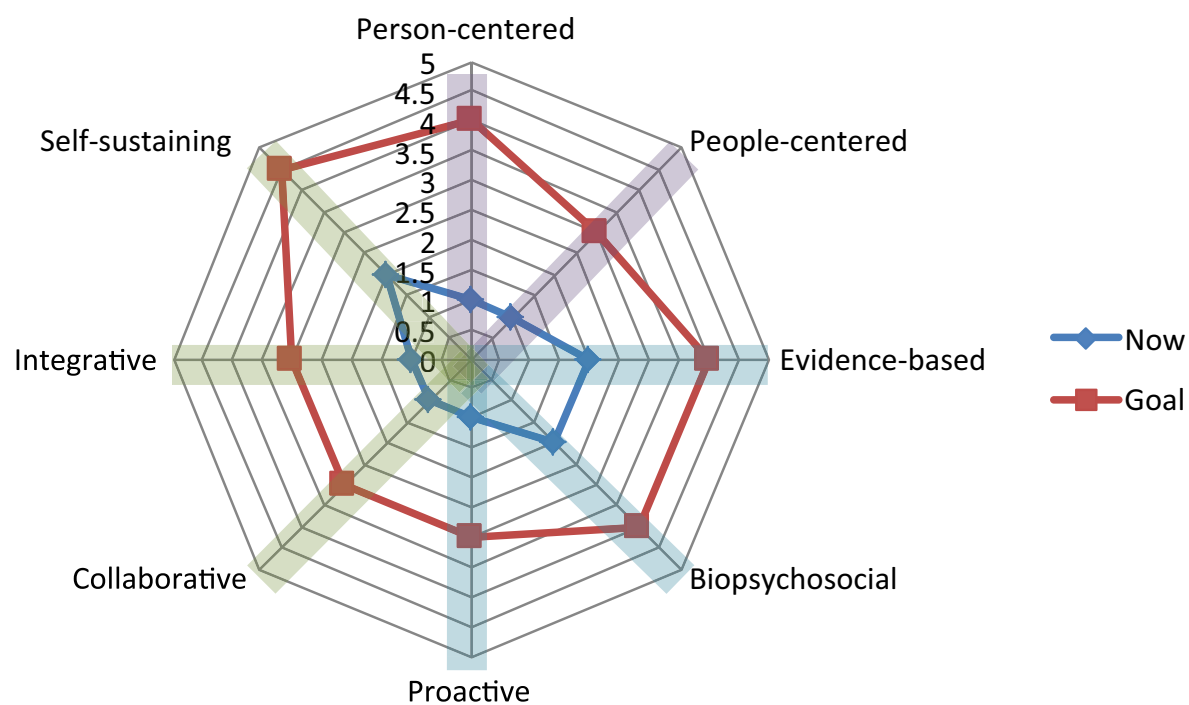

Purple $=$ who is the focus of the care?

Blue $=$ what type of care is given by health care provider?

Green $=$ in what manner is spine care delivered? 
care levels including secondary and tertiary centers that provide advanced spine care.

Self-care and community spine care programs

A majority of people do not need medical treatment but instead could benefit from education and reassurance. Therefore, we propose programs and policies that support prevention, healthy behaviors, and safe environments in the home, work, and play. Public health education and community-based programs can educate the population about factors that increase the likelihood of spine-related disability. On an individual level (e.g., patient or caregiver), the public media (e.g., internet, television, radio, social media) or encounters with health care providers can deliver educational campaigns, such as for exercise and prevention [72]. Community-based programs should address population needs, such as screening programs for osteoporosis or prevention programs to reduce traumatic spine injury.

Primary spine care

Most people who could benefit from health care interventions have spinal disorders that can be adequately managed at a primary care level. The World Health Organization defines primary care as "first-contact, accessible, continued, comprehensive, and coordinated care to people and communities" [73]. Primary care may provide beneficial and cost-effective care for some spinal conditions [74-78] and is the point of entry for the majority of spine care, excluding trauma and urgent care, and plays an important gatekeeping role, including triage, diagnosis and referral of patients so people receive appropriate care as early as possible [79]. We propose that primary care for spine-related concerns should focus on common spinal conditions using non-invasive, active, and patient-driven interventions for spine-related complaints, including prevention and rehabilitation [80-88].

Primary spine care services may be provided in different ways depending on available resources [89]. A single clinician may suit the needs at one location, whereas in another location that might not have a primary care provider with all the necessary competencies, a team of clinicians, when combined, could have the knowledge and skills to provide primary spine care. Different provider types have the potential to offer primary spine care depending on their education (e.g., medical doctors, doctors of osteopathy, doctors of chiropractic, physical therapists, physician assistants, nurse practitioners, other clinicians) $[74,80-84,90]$. Those who do not yet have the necessary skills and knowledge may be

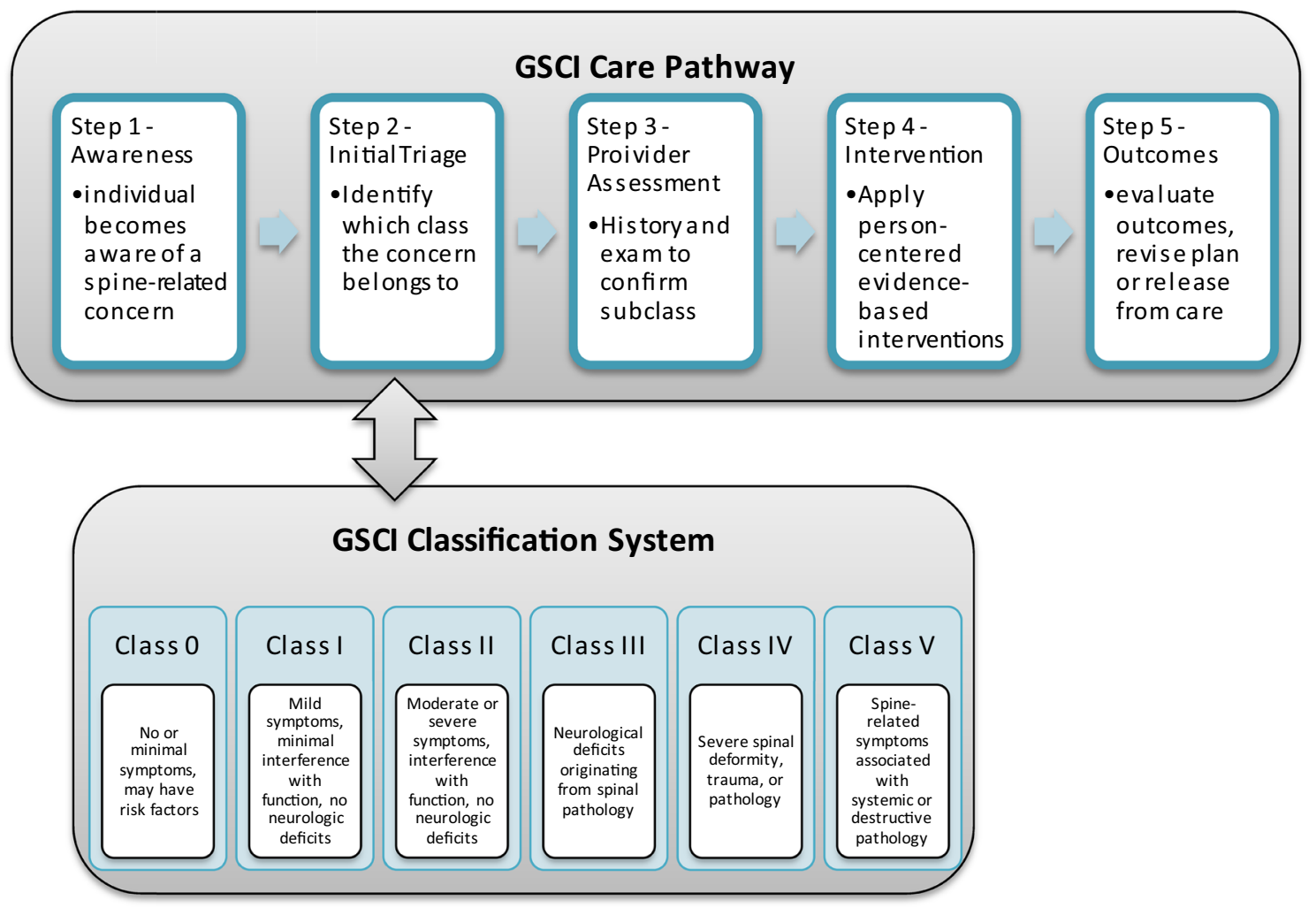

Fig. 3 An overview of the GSCI Care Pathway [34]. This care pathway guides health care practitioners through a short series of steps to help provide best spinal care. The GSCI classification system [33] allows community health workers or health care practitioners to quickly and easily classify people who present with spine-related concerns. The steps of the care pathway are integrated within the health care system to improve access, reduce cost, and increase quality of spine care 

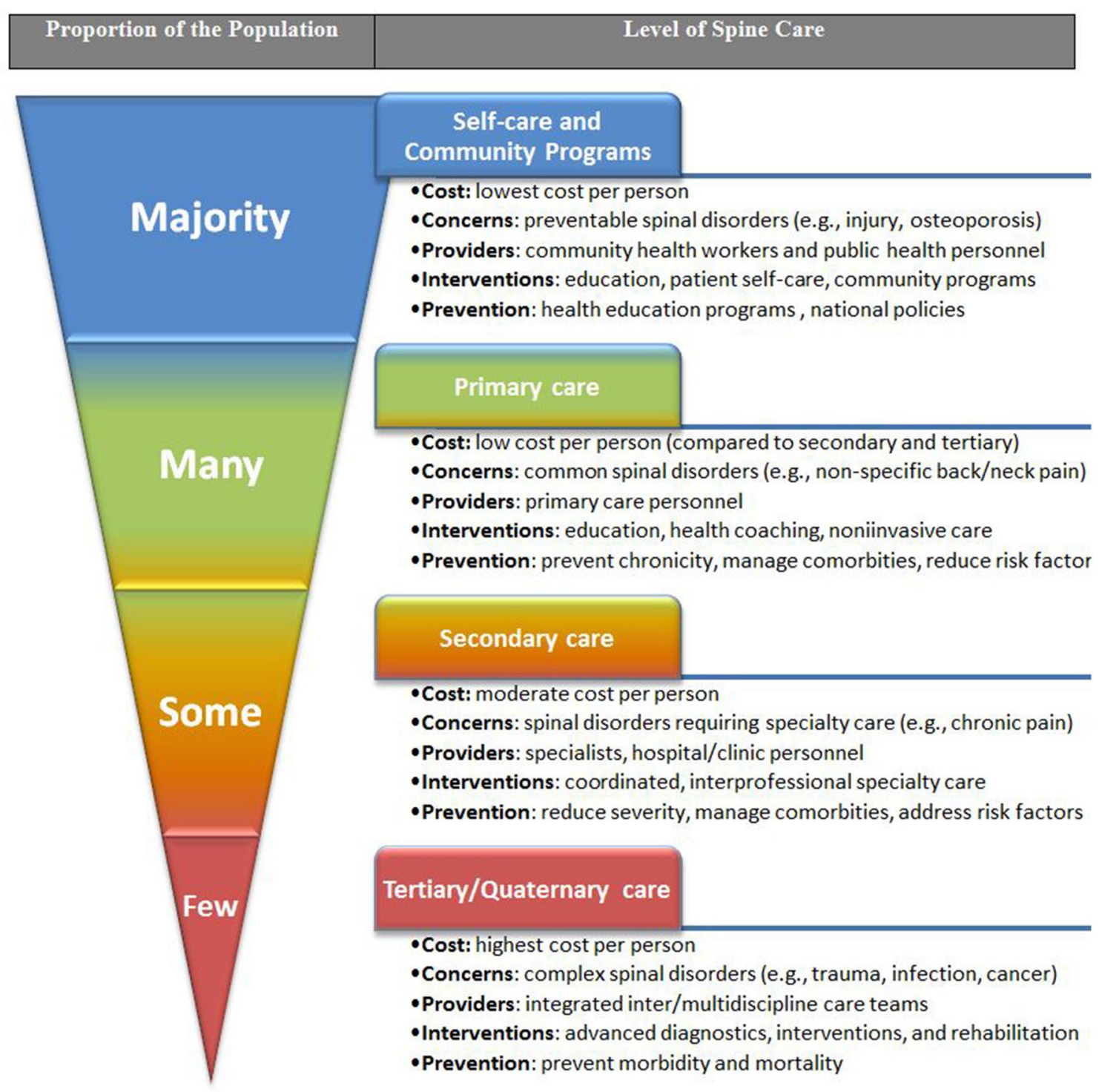

Fig. 4 Levels of care in the GSCI model of care. Reproduced with permission from World Spine Care

trained to offer spine care. Some providers with limited general health care training, such as community health workers or traditional healers, may also be included as team members for spine care, thus expanding feasibility to offer primary spine care [91-93]. To function as a primary spine care provider or primary spine care team, the providers should have a professional health care degree recognized by the local or national government and the necessary training, knowledge, and skills to deliver care in the care pathway. We recommend a list of primary spine care provider competencies [3, 29-32, 38] (see Online Resource Appendix 1).

Primary spine care must be integrated into the health care system as it is responsible for coordinating the care for complex disorders that may require interventions at the secondary and tertiary spine care settings. Primary spine care is responsible for offering continuous and coordinated care throughout the patient's condition. Patients may require treatment for comorbidities or health conditions that are related to, but not generated from the spine; thus, referral to care providers in other areas may be required. The primary spine care provider/team should develop an established protocol to give and receive referrals with other primary care physicians (family practice, internal medicine, pediatrics, obstetrics-gynecology) and secondary or tertiary spine care for specialty services (e.g., psychological care, physiatry, pain management, rheumatology, surgery, other invasive therapies, advanced pharmacologic therapy, multidisciplinary team care) and community health programs. Primary spine care services need to be connected and communicated to the community through: 1) community-based programs 
that educate about prevention of spine-related conditions and disability; 2) provision of educational programs for individuals on self-care and prevention; and 3) screening and education on risk factors or comorbidities associated with spine symptoms (e.g., osteoporosis/tuberculosis screening, tobacco cessation programs, avoidance of opioid or other drug dependencies)

Secondary spine care

Only some people have spinal conditions that require more advanced care. Secondary spine care is care provided by one or more medical specialists, typically at the request of the primary care provider. Secondary services may include shortterm interventions including consultation, hospitalization, or treatments such as advanced pharmaceuticals, injections, individualized rehabilitation protocols, or surgery. Secondary spine care should include specialized cognitive-behavioral interventions to address any inappropriate pain behaviors and disability, in particular work disability. Secondary spine care specialists and teams work collaboratively with other health care providers; thus, a close relationship between secondary and primary care spine providers assists with rehabilitation and conservative post-operative care or in follow-up to secondary care interventions. Secondary care providers send and receive referrals from primary and tertiary care according to the care pathway. Any person who initially presents to secondary care but who should be receiving primary care should be rerouted to a primary care provider.

Tertiary/quaternary spine care

Very few people have spinal conditions that require tertiary or quaternary care. Tertiary spine care, where available, is specialized medical and surgical care for complex and unusual spine problems. Tertiary spine care provides complex invasive spinal treatments including advanced pharmaceuticals and surgery, requiring an interdisciplinary or multidisciplinary health care team. Tertiary spine care teams work collaboratively with others in the health care system. They send and receive referrals from primary and secondary care according to the care pathway. Providers in tertiary and quaternary spine care work collaboratively with other health care providers including secondary and primary care. Quaternary care is extremely rare, highly specialized and may be experimental, thus unlikely to be found in lowincome countries or underserved areas. We recognize that some regions do not readily have access to either tertiary or quaternary care.

\section{GSCl person's journey}

The GSCI person's journey provides a visual diagram of how someone with a spine-related concern might navigate the GSCI model of care. This map helps communicate with stakeholders (patients, community members, health care providers, decision makers, payers, etc.) so they may visualize how a person will journey through the model of care (See Fig. 5). The GSCI model of care approaches care delivery by focusing on how an individual may perceive or search for care and answers to spine-related questions. Care should be delivered based upon the local culture and within the available resources. The spine care provider considers initial information that is presented and triages the patient according to current evidence-based guidelines and recommendations of the GSCI classification and pathway. Interventions range from self-care and community-based education through more advanced care when indicated.

\section{GSCI model of care implementation}

We propose six steps that should be considered when deploying the GSCI model of care. Each community and setting is unique; therefore, the implementation plan will need to be customized. Some steps may need to be expanded, modified, or even skipped depending upon the spine care needs of a community, available resources, and how similar or dissimilar the current system is to the model of care. The timeline for implementation will vary as it is dependent on the given situation and will need to be customized for each location.

Step 1 Project initiation and initial preparations

1. Identify a community whose members want and need a spine care program [40]. A community may self-identify or an external entity may identify a community as a potential candidate.

2. Define and prioritize the spine-related issues for that community. Perform a preliminary review of the spinerelated concerns, burdens, facilitators, and barriers [44, 46, 94-97]. These baseline measurable outcomes will shape implementation and measure progress. Examples may include: number of work days lost due to spine pain or injury, cost of care for a given spinal condition, or number of people who have or develop a spinal condition.

3. Assess if key stakeholders and decision leaders are interested and able to establish a spine program. Obtain preliminary information, such as location/region, population, health care delivery system, level of human resources, infrastructure, funding mechanisms, and specific needs help determine feasibility.

4. Once a clear need has been identified and the capacity for change appears feasible, develop a "case for change" statement. This statement should clearly describe the need, goals, and scope of the project [98]. Use this vision statement when communicating with others about the project. The "case for change" will help to ground the pathway of change, such as through using a logic 


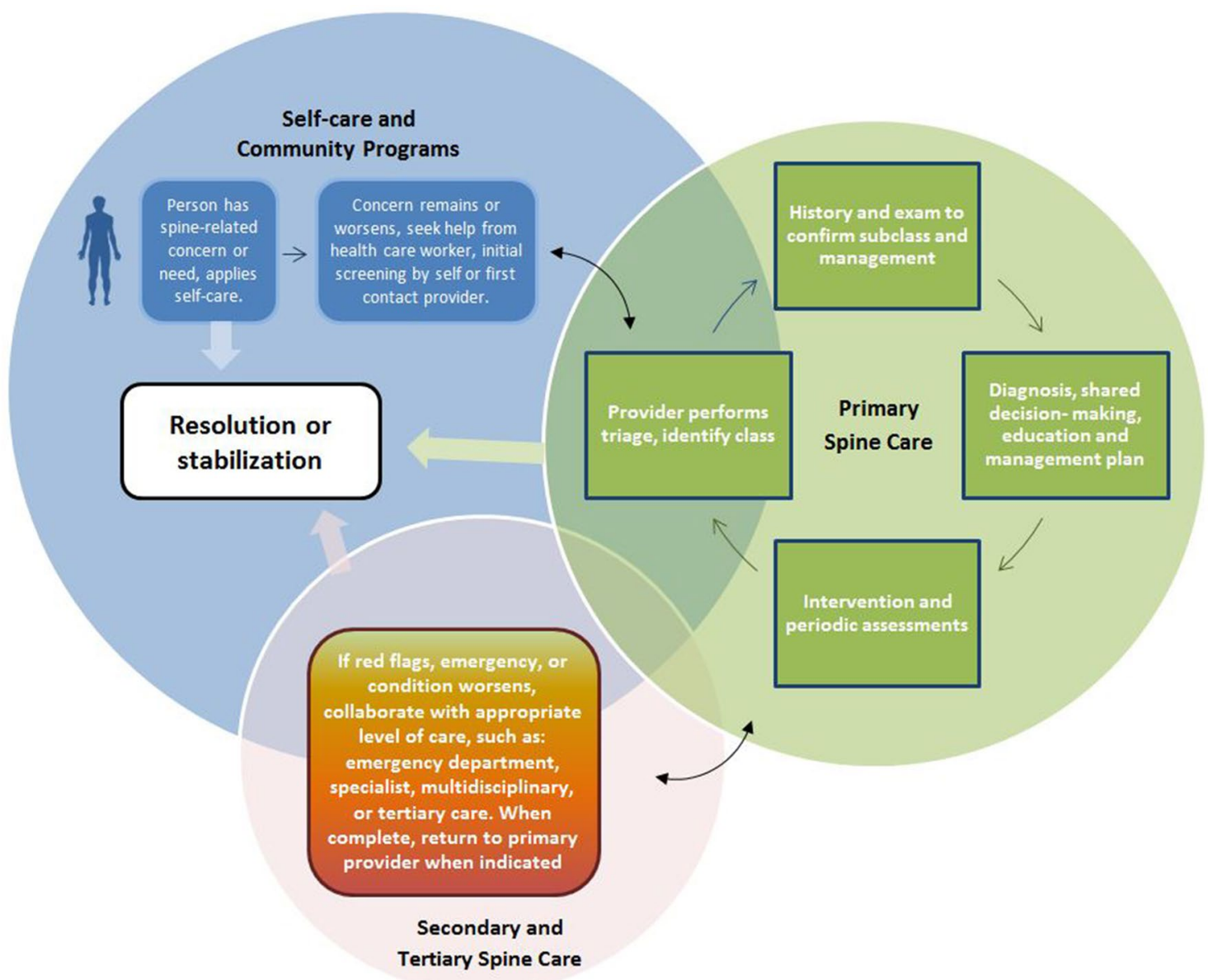

Fig. 5 Model of care—diagram of a person's journey. Reproduced with permission from World Spine Care

model. (See Online Resources Figure 2 for an example of a logic model). Share the vision of the program and obtain agreement and participation from leadership. Support from decision makers (executives, clinical champions) is essential since change is unlikely if recognized leaders are not involved in the decision-making process.

5. Meetings should take place between key stakeholders to evaluate the assessments and to weigh benefits and barriers to implementation [99]. A final decision should be made regarding if the project appears feasible and if it should begin.

6. After it is agreed that the project should begin, obtain support from additional stakeholders, such as those receiving care (patients and community), those providing care (community health workers and providers, health care clinics, hospitals), and those funding the care. Additionally, consider including those who are thought leaders within the community, such as traditional healers and religious leaders.
7. Establish the steering committee and the implementation team [99].

a. Steering committee The steering committee directs the course of action at a high level. This committee invites the stakeholders who have the authority to make changes and are relevant to the success of the project to be members of the steering committee, such as stakeholders who are decision makers, who provide input, and who may be affected by the program. The steering committee decides on the overarching goals, objectives, and scope of the project based upon available local health information and estimated needs. These initial goals should be locally relevant and all stakeholders on the steering committee should agree. (See Online Resource Appendix 2 for sample checklists)

b. Implementation team The steering team selects implementation team members who are the local stakeholders involved with planning and implement- 
ing the model and have the knowledge and skills to implement the plan on a day to day level. The steering committee and the implementation team have different members, but they could overlap. The strategic planning members continue to work with the implementation team through the entire process.

Step 2 Assessment of the current situation

1. Before making any changes, the implementation team must first assess the current health care system (infrastructure, resources, funding, processes, personnel, and clients (individuals and community)) to identify needs related to spine care. This information helps establish baseline resources and further informs the implementation process before the program can be implemented [100]. Consider using or modifying a checklist throughout this process. The following are actions to consider in the assessment step.

a. For the location that you will implement the model of care, use the Model of Care Principles Transition Tool to measure the eight principles: person-centered, people-centered, biopsychosocial, proactive, evidence-based, integrative, collaborative, and selfsustaining.

b. Assess the community and individuals' values and needs as they relate to spinal disorders, health, and care, including knowledge about spinal disorders, risk factors and comorbidities, population demographics, access to care, and social factors (e.g., religion, culture, and language) [101].

c. Determine roles of participants, including patients, family, health care providers/staff, community, health care system [102,] and other participants such as funders and collaborators. During assessment, consider establishing collaborative connections between health and other sectors that influence success of the program.

d. Assess the local health care system resource capacity required to implement the desired spine care program. This helps to determine what resources will need to be developed (e.g., human resources) or obtained (e.g., materials) [3]. Also assess if current resources are being used effectively.

e. Identify processes and how spine care is currently being delivered. Assess the gap in behaviors, processes [103], and resources between the current model and the new model of care, such as using the GSCI classification system and care pathway to see what type of gap must be closed before implementation. f. Establish a list of resources and processes that are needed to implement the model of care.

g. Identify barriers and facilitators in the local community. If possible, use a theoretical framework to address barriers and facilitators. Select evidence from the literature that will support the steps in the plan for change [103]. Barriers and facilitators vary in types and complexity and are unique to each local community thus should be addressed in implementation plans.

h. Assess policies, systems, and other available resources in the target community. Identify if professional, educational, and health care policies are able currently to support implementation or if policy updates are needed [102].

i. Identify determinants of change in the current health care system to implement or transition to a new model, which includes barriers and facilitators. Assess health care system readiness and what may be needed locally to optimize sustainability [40].

Step 3 Planning and designing solutions

1. After reviewing the initial assessment, the steering committee provides direction and the implementation team then develops the plan based upon information from the assessments. Implementing evidence-based models of care can be challenging, especially in low- and middleincome countries [95]. Multifaceted strategies are effective, whereas single faceted strategies are unlikely to be adopted or make a change [95]. Thus, a clear plan with multifaceted strategies may be helpful. Include health care providers in planning and provide training program so they may learn or be refreshed on the evidence-based care pathway. Those who are not directly engaged but were identified in the process assessment are included so that they may properly refer or collaborate with the process. Capacity building begins during planning but continues through the implementation, evaluation, and scaling up. Once the plan has been drafted, invite additional stakeholders to review the plan to provide input and feedback. The following are items to include in the plan.

a. Include the guiding principles in the implementation plan (e.g., person-centered, people-centered, biopsychosocial, proactive, evidence-based, integrative, collaborative, and self-sustaining) as they relate to local needs [98].

b. Clearly describe the steps needed to transform the current system in preparation for implementation of the spine program for each of the principles, so that 
stakeholders and those doing the implementation can understand the process and will know what to do. Each plan will be unique based upon the current resources and needs of the community.

c. Include the plan for capacity building (e.g., training health care workers to use the GSCI model of care, securing resources), budget, evaluation process, identifying key performance indicators, short, intermediate, long range goals, and other essential items, which may include a memorandum of understanding with decision makers and infrastructure issues [104]. If not already in existence, consider adapting tools to local language and culture [105]. Using well-known planning tools, such as logic models are recommended [100]. (See Online Resources Figure 2 for an example of a logic model).

d. Establish evaluation measures for key performance indicators, whether this includes modification of an existing evaluation system or the adoption or creation of a new system and use of new measures. Evaluation plans should be focused to efficiently assess the most important concerns of the stakeholders [100].

e. Identify outcome measures to assess major components and principles of the model and the processes by which they will be measured. Include outcomes such as metrics focused on the patient (values, function, activities), population health outcomes, costs/ cost savings, and value of care indicators, biopsychosocial metrics, program success, and sustainability metrics. Patient outcomes, satisfaction by clinical, staff and an assessment of community acceptance will demonstrate improvement, identify the strengths and weaknesses, and allow for modification during implementation.

f. Describe and outline the reporting structure and how the participants and the system will be held accountable. Incorporate into the plan collaborative relationships, communication systems, educational programs to prepare the health care workforce, and funding sources.

g. Describe how you will recruit and train local community health workers and qualified primary spine care providers to use the GSCI model of spine care. Describe the preparation of workforce, infrastructure, and sustainability factors (funding) to support the implementation of the model of care. Describe steps to educate and do outreach to health care providers and the community to prepare for the implementation of the model of care.

h. Include key components, such an explicit list of steps with who is responsible for each step, a time- line (e.g., Gantt chart), budget, and communications strategy.

i. Develop a communication plan and prepare the existing health system and community for the upcoming program implementation $[99,100]$.

j. The final action in this step should be to have key stakeholders review the implementation plan before moving to the next step.

\section{Step 4 Implementation}

1. Perform an initial small scale implementation.

a. Once the steering committee and key stakeholders have approved the plan, the implementation team should complete a small level rollout. This will help demonstrate effective implementation. This initial implementation step builds confidence with the stakeholders and informs the steering committee and implementation team if the system is ready for more substantial scale implementation.

b. Include all components and assessments but on a small scale. Because the initial implementation will test the system, the pilot should incorporate all other components to test the full system in addition to the implementation of care.

c. Include collecting outcome measures in the pilot.

d. Evaluate the pilot both formative and summative manners, which will help inform the steering committee what additional resources or assistance is needed to implement the complete model of care. Because each location is unique, successful implementation requires flexibility and demonstration of proof of concept before initiating the larger scale rollout.

e. Revise and update the implementation plan based on the information from the pilot.

2. Full implementation. Once the steering committee and implementation team agree that full implementation is feasible, commence the implementation plan for a full scale rollout.

Step 5 Assessment and evaluation of program

1. Assessment and evaluation

a. Collect outcome measures for levels that were established during the planning stage, including the eight principles.

b. The steering committee and implementation team should evaluate the data to identify strengths and weaknesses, identify areas of potential improve- 
ment, to see if program goals are being met, what course corrections are needed, and if any new goals need to be introduced.

c. Consider continuing to use the Model of Care Principles Transition Tool to identify if the program is meeting its original goals.

d. On a regular basis, the team should review the data and then develop a plan to make improvements based on the data. These steps are part of the continuous quality improvement process ("plan do study act" cycle), which will evaluate the key performance indicators at regular intervals and inform further direction of change from the old to the new model of care. Key performance indicators inform program sustainability [100]. A continuous quality improvement cycle is essential to improving the program to reach the community health care goals [95]. During evaluation, updates are made to the course or plan to improve and maintain initiatives. Further plans are made to implement the improvements and to better maintain the program.

e. Once the steering committee feels the program is sustainable, future evaluations may include action research if resources are available.

f. The steering committee should communicate evaluation findings to stakeholders.

Step 6 Sustain program and scale up

1. Sustain program

a. Monitor the program to ensure that support, resources, communications, and ongoing training will continue.

b. Use ongoing continuous quality improvement measures to make sure that the program stays on course and addresses evolving community spine care needs.

c. Engage with stakeholders. Share information about long-term benefits and impact of implementation of the model of care [102].

d. Ensure that leaders are committed to sustaining the program. Provide access to training in sustaining programs, if possible [100].

2. Scaling up

a. Once the program has demonstrated success at a local level, scaling up should be considered [106, 107]. Scaling up means "doing something in a big way to improve some aspect of a population's health and requires sustainability, equity and the effects of scaling up an intervention (or a package of interventions) on the rest of the health system (exter- nal consequences)" [108]. Scaling up is taking a smaller-scale successful initiative and implementing it on a larger scale. This could mean expanding the program on a local level, expanding nationally, or bringing the program to other regions.

\section{Discussion}

The primary result of this study was the development of the GSCI model of care for spinal disorders. The model of care was informed by GSCI literature reviews and the public health literature. An extensive panel of international experts distilled the values of the model of care down to eight core principles. Based on these principles, the model can be linked to a six-step implementation plan. We believe that the proposed GSCI model of care is the first of its kind to address the global burden of spinal disorders and recent calls for action [7, 23, 109].

The WHO defines high-quality care as "care that is safe, effective, people-centred, timely, efficient, equitable and integrated" [110]. The aim is to maximize health outcomes, prevent disability, and reduce costs and we feel that the GSCI model addresses these issues. A central component is the inclusion of primary spine care. However, it is important not to over-simplify primary care in low-resource settings. In some communities, primary care is the only level of care available and may be overburdened with urgent and lifethreatening problems and may have no other health care support structure. As stated by the World Health Report (2008) "it is not acceptable that in low-income countries, primary care would be reduced to a stand-alone health post or isolated community-health worker" [111]. The WHO aims to have primary care in low-resource areas include the capacity to manage and refer a range of health problems, help guide a patient through the health system, include shared decision making and patient-centered practices, apply prevention and health promotion, team of providers with biomedical and social skills, and be accessible and affordable [111]. This primary care based system and the recommendation to shift toward outpatient and ambulatory care is supported by the WHO global strategy [57]. As well, the United Nations have set out the 2030 Sustainable Development Goals that provides a list of targets to achieve across all nations [112]. Many of the GSCI principles are in alignment as they relate to health, well-being, access to care, and implementation.

Spine care practices must be relevant locally. Practices that are efficient in a high-income country may not necessarily be applicable in communities with scarce resources or in cultures that are dissimilar. As well, there are different approaches to how health care systems can be organized in various countries [113]. Local health care infrastructure 
varies and may include a traditional healer as the sole provider of care or instead be provided through community clinics, district, or tertiary hospitals. Each of these environments has unique ways to approach spine care. Within these, there can be cultural, financial or other barriers to implementation. Therefore, we chose a broad approach to this model of care. Thus, the description is non-specific regarding what type of health care system the model could be implemented in because our goal was to develop a model that was flexible and could be applied in most health care systems and countries.

Another valuable asset of the proposed model is that any provider (i.e., recognized by the local government) who has the knowledge and skills as described in this paper should be able to engage and implement the GSCI model of care. This is especially helpful in regions with few resources. Because each world region has different cultures, access to resources, and different providers, we chose not to make the model center around one health care profession or discipline. This allows the greatest application of the model in the greatest number of regions. As well, components of the proposed model may be easily taught to clinicians and stakeholders by using visual educational tools (e.g., chart or flashcards) and the classification and care pathway may be easily adapted into electronic medical record software.

Improving or changing current health care practices and systems can be challenging [94]. Even if an individual provider is able and willing to change, the health care leadership, political environment, local culture, and community members must also be willing to engage and participate. Because of its complex nature, a planned implementation to address multiple factors is required. A combination of tools and strategies that may be effective in one location may not necessarily be so in another due to unique characteristics, barriers, and facilitators. Thus, the implementation plan for the model of care for each location must be crafted to address these unique factors. However, basic steps in implementation may help to facilitate program success. In some low-income communities, it may be that one small step is implemented and then over time, other steps will follow. In locations with more robust resources, infrastructure can be built earlier and more rapidly. The process must be uniquely individualized to community needs and available resources.

Testing a new model of care is a vibrant process in which we expect there to be both successes and failures. Because the GSCI model of care includes assessment and evaluation measures, it is expected that the successes will outweigh the failures since continuous quality improvement and course correction are built into the model. We feel that adaptability is one of the most valuable characteristics of the GSCI model. What matters most is that the needs of the community are addressed and the long range goals are kept in the forefront of the minds of leaders and stakeholders.

\section{Limitations}

As with any process used to develop a new model, there are limitations. First, efforts to develop a model that could be applied in any community, especially underserved regions and low- and middle-income countries, were especially challenging. Even though there is a recognized need, there is little available and reliable information about this topic in the literature. What information is available mainly comes from high-income countries and these are often burdened by the principles we are recommending we transition away from. We hope that the application of this new model can be tested in various regions so that those working to improve spine care may collect information and gain wisdom from its application. Also, not everyone we invited was able to participate. Therefore, the model is limited by the thoughts of the expert team of people who contributed to it. As with any model, this one needs to be tested in the field before the value of the model can be critiqued. What may be successful in one location may not necessarily be so in another.

\section{Conclusion}

A panel of international spine care experts developed the GSCI model of care based on eight core principles. The aim of this model is to help transform spine care globally, but especially in low- and middle-income regions and underserved communities. We consider this paper as a first step in a dialogue about this proposed model. It is our expectation that the GSCI model is dynamic and will evolve with new evidence and its application. We welcome others to join in our efforts, along with critiques, suggestions, and implementation of this model aimed at improving spine care globally.

Funding The Global Spine Care Initiative and this study were funded by Grants from the Skoll Foundation and NCMIC Foundation. World Spine Care provided financial management for this project. The Funders had no role in study design, analysis, or preparation of this paper.

\section{Compliance with ethical standards}

Conflict of interest CDJ is president of Brighthall Inc; she is an NCMIC Board of Director; however, neither she nor NCMIC board make funding decisions for the NCMIC Foundation; the views in this article are those of the authors and not those of Stanford University, Stanford Health Care, or Qualcomm. SH declares funding to UOIT from Skoll Foundation, NCMIC Foundation through World Spine Care. Clinical Policy Advisory Board and stock holder, Palladian Health. Advisory Board, SpineHealth.com. Book Royalties, McGraw Hill. Travel expense reimbursement-CMCC Board. RC declares funding from AHRQ to conduct systematic reviews on treatments for low back pain within last 2 years. Honoraria for speaking at numerous meetings of professional societies and non-profit groups on topics related to low back pain (no industry sponsored talks). MN declares fund- 
ing from Skoll Foundation and NCMIC Foundation through World Spine Care; Co-Chair, World Spine Care Research Committee. Palladian Health, Clinical Policy Advisory Board member. Book Royalties Wolters Kluwer and Springer. Honoraria for speaking at research method courses. BNG receives speaker fees and travel reimbursement from NCMIC Speakers' Bureau; he is secretary of Brighthall Inc; the views in this article are those of the authors and not those of Stanford University, Stanford Health Care, or Qualcomm. PC is funded by a Canada Research Chair in Disability Prevention and Rehabilitation at the University of Ontario Institute of Technology, and declares funding to UOIT from Skoll Foundation, NCMIC Foundation through World Spine Care. Canadian Institutes of Health Research Canada. Research Chair Ontario Ministry of Finance. Financial Services Commission of Ontario. Ontario Trillium Foundation, ELIB Mitac. Fond de Recherche and Sante du Quebec. EH declares he is a consultant for: RAND Corporation; EBSCO Information Services; Southern California University of Health Sciences; Western University of Health Sciences Data and Safety Monitoring Committee. Chair, Palmer Center for Chiropractic Research. Research Committee Co-chair, World Spine Care. DKG declares travel expenses: CMCC to present at the WSC Spine Conference in Botswana. EAc declares grants: Depuy Synthes Spine, Medtronic; Speaker's bureau: AOSpine, Zimmer Biomet. KR declares funding to UOIT from Skoll Foundation, NCMIC Foundation through World Spine Care. EA declares funding to UOIT from Skoll Foundation, NCMIC Foundation through World Spine Care. TB declares European Spine Journal provided a grant to investigate scoliosis. OB declares he is a consultant for: Pacira Pharmaceuticals, Inc. Palladian Health. Travel expenses: World Spine Care. Stipend: World Spine Care. PB declares contribution to salary for Global Musculoskeletal Alliance (G-MUSC), The Bone and Joint Decade work. JMC declares Government Grant ESPY 1281/15. SE is an employee of the Botswana Ministry of Health and Wellness. CG declares travel expenses: Palmer College to GSCI meetings. Consultant: American Chiropractic Association, Spine IQ, Healthwise, Quality Insights of Pennsylvania, RAND Co.; Prezacor, Inc. (Stock Options). PCORI (Board Member). Grants: Collaborative Care for Veterans with Spine pain and Mental Health Issues. NIH/Kiernan Chiropractic Care in Rehabilitation at Crotched Mountain: Crotched Mountain Private Sector Integrated Chiropractic Study N/A. NCMIC Foundation Chiropractic services, Assessment of Chiropractic Treatment for Low Back Pain; RAND Subcontract, Department of Defense Prime Award \#W81XWH-11-2-017 Sub \#9920110071. JH declares his research group has extensive Funding from Danish public Funding agencies, the European Union, and Danish charities. MH declares travel support from World Spine Care. JM declares general research resources from USF Research Center. Research Grants from funding agencies: FEMA, US Department of Homeland Security (EMW-2013-FP-00723). Palladian Health Advisory board: Clinical Policy and Advisory Board. Intellectual property rights: Inventor of Web-based system to deliver exercise (Employer-USF: copyright holder). TM declares Fellowship GrantMedtronics. JM declares WSC Board Member. EM declares AO Spine Africa Faculty courses-honorarium. GO declares he is a consultant and receives travel support as Clinic Director, World Spine Care. HR declares Funding to UOIT from Skoll Foundation, NCMIC Foundation through World Spine Care. MS declares he is a Scientific Advisor, NuSpine; Consultant, and State Farm. ES declares funding from Başkent University Research Fund. ATV declares funding to UOIT from Skoll Foundation, NCMIC Foundation through World Spine Care. LV declares funding to UOIT from Skoll Foundation and NCMIC Foundation through World Spine Care. WW declares Palladian Health, Clinical Policy Advisory Board member. JW declares funding to UOIT from Skoll Foundation, NCMIC Foundation through World Spine Care. HY declares funding to UOIT from Skoll Foundation, NCMIC Foundation through World Spine Care and the remaining authors declare that they have no conflict of interest.
Open Access This article is distributed under the terms of the Creative Commons Attribution 4.0 International License (http://creativeco mmons.org/licenses/by/4.0/), which permits unrestricted use, distribution, and reproduction in any medium, provided you give appropriate credit to the original author(s) and the source, provide a link to the Creative Commons license, and indicate if changes were made.

\section{References}

1. Hurwitz EL, Randhawa K, Torres P, Yu H, Verville L, Hartvigsen J et al (2017) The Global Spine Care Initiative: a systematic review of individual and community-based burden of spinal disorders in rural populations in low- and middle-income communities. Eur Spine J. https://doi.org/10.1007/s00586-017-5393-z

2. Hurwitz EL, Randhawa K, Yu H, Cote P, Haldeman S (2018) The Global Spine Care Initiative: a summary of the global burden of low back and neck pain studies. Eur Spine J. https://doi. org/10.1007/s00586-017-5432-9

3. Acaroglu E, Nordin M, Randhawa K, Chou R, Cote P, Mmopelwa T et al (2018)The Global Spine Care Initiative: a summary of guidelines on invasive interventions for the management of persistent and disabling spinal pain in low- and middle-income communities. Eur Spine J. https://doi.org/10.1007/s0058 6-017-5392-0

4. Buchbinder R, Blyth FM, March LM, Brooks P, Woolf AD, Hoy DG (2013) Placing the global burden of low back pain in context. Best Pract Res Clin Rheumatol 27(5):575-589

5. Briggs AM, Woolf AD, Dreinhöfer K, Homb N, Hoy DG, Kopansky-Giles D et al (2018) Reducing the global burden of musculoskeletal conditions. Bull World Health Organ 96(5):366

6. Hay SI, Abajobir AA, Abate KH, Abbafati C, Abbas KM, AbdAllah F et al (2017) Global, regional, and national disabilityadjusted life-years (DALYs) for 333 diseases and injuries and healthy life expectancy (HALE) for 195 countries and territories, 1990-2016: a systematic analysis for the Global Burden of Disease Study 2016. The Lancet 390(10100):1260-1344

7. Hartvigsen J, Hancock MJ, Kongsted A, Louw Q, Ferreira ML, Genevay S et al (2018) What low back pain is and why we need to pay attention. The Lancet 391:2356-2367

8. Dagenais S, Caro J, Haldeman S (2008) A systematic review of low back pain cost of illness studies in the United States and internationally. Spine J 8(1):8-20

9. Whitehurst DG, Bryan S, Lewis M, Hill J, Hay EM (2012) Exploring the cost-utility of stratified primary care management for low back pain compared with current best practice within risk-defined subgroups. Ann Rheum Dis 71(11):1796-1802

10. Spiegel DA, Gosselin RA, Coughlin RR, Joshipura M, Browner BD, Dormans JP (2008) The burden of musculoskeletal injury in low and middle-income countries: challenges and opportunities. J Bone Jt Surg Am 90(4):915-923

11. Beveridge M, Howard A (2004) The burden of orthopaedic disease in developing countries. J Bone Jt Surg Am 86-A(8):1819-1822

12. Joshi R, Alim M, Kengne AP, Jan S, Maulik PK, Peiris D et al (2014) Task shifting for non-communicable disease management in low and middle income countries-a systematic review. PLoS ONE 9(8):e103754

13. Mody GM, Brooks PM (2012) Improving musculoskeletal health: global issues. Best Pract Res Clin Rheumatol 26(2):237-249

14. Punchak M, Mukhopadhyay S, Sachdev S, Hung Y-C, Peeters S, Rattani A et al (2018) Neurosurgical care: availability and access 
in low-income and middle-income countries. World Neurosurg 112:e240-e254

15. Edwards J, Hayden J, Asbridge M, Gregoire B, Magee K (2017) Prevalence of low back pain in emergency settings: a systematic review and meta-analysis. BMC Musculoskelet Disord 18(1):143

16. Moradi-Lakeh M, Forouzanfar MH, Vollset SE, El Bcheraoui C, Daoud F, Afshin A et al (2017) Burden of musculoskeletal disorders in the Eastern Mediterranean Region, 1990-2013: findings from the Global Burden of Disease Study 2013. Ann Rheum Dis annrheumdis-2016-210146

17. Riley L, Guthold R, Cowan M, Savin S, Bhatti L, Armstrong T et al (2016) The World Health Organization STEPwise approach to noncommunicable disease risk-factor surveillance: methods, challenges, and opportunities. Am J Public Health 106(1):74-78

18. Abegunde DO, Mathers CD, Adam T, Ortegon M, Strong K (2007) The burden and costs of chronic diseases in low-income and middle-income countries. The Lancet 370(9603):1929-1938

19. Geneau R, Stuckler D, Stachenko S, McKee M, Ebrahim S, Basu $S$ et al (2010) Raising the priority of preventing chronic diseases: a political process. The Lancet 376(9753):1689-1698

20. Alwan A, MacLean DR, Riley LM, d'Espaignet ET, Mathers CD, Stevens GA et al (2010) Monitoring and surveillance of chronic non-communicable diseases: progress and capacity in high-burden countries. The Lancet 376(9755):1861-1868

21. Strong K, Mathers C, Leeder S, Beaglehole R (2005) Preventing chronic diseases: How many lives can we save? The Lancet 366(9496): 1578-1582

22. Hoy D, March L, Brooks P, Blyth F, Woolf A, Bain C et al (2014) The global burden of low back pain: estimates from the Global Burden of Disease 2010 study. Ann Rheum Dis 73(6):968-974

23. Buchbinder R, van Tulder M, Öberg B, Costa LM, Woolf A, Schoene $M$ et al (2018) Low back pain: a call for action. The Lancet 391:2384-2388

24. Johnson CD, Haldeman S, Nordin M, Chou R, Côté P, Hurwitz EL et al The Global Spine Care Initiative: methodology, contributors, and disclosures. Eur Spine J. https://doi.org/10.1007/ s00586-018-5723-9

25. Diamond IR, Grant RC, Feldman BM, Pencharz PB, Ling SC, Moore AM et al (2014) Defining consensus: a systematic review recommends methodologic criteria for reporting of Delphi studies. J Clin Epidemiol 67(4):401-409

26. Hsu C-C, Sandford BA (2007) The Delphi technique: making sense of consensus. Pract Assess Res Eval 12(10):1-8

27. Dalkey NC (1969) The Delphi method: an experimental study of group opinion. RAND Corp Santa Monica, CA

28. Acaroglu E, Mmopelwa T, Yuksel S, Ayhan S, Nordin M, Randhawa K et al (2017) The Global Spine Care Initiative: a consensus process to develop and validate a stratification scheme for surgical care of spinal disorders as a guide for improved resource utilization in low- and middle-income communities. Eur Spine J. https://doi.org/10.1007/s00586-017-5332-z

29. Ameis A, Randhawa K, Yu H, Cote P, Haldeman S, Chou R et al (2017) The Global Spine Care Initiative: a review of reviews and recommendations for the non-invasive management of acute osteoporotic vertebral compression fracture pain in low- and middle-income communities. Eur Spine J. https://doi.org/10.1007/ s00586-017-5273-6

30. Cedraschi C, Nordin M, Haldeman S, Randhawa K, KopanskyGiles D, Johnson CD et al (2018) The Global Spine Care Initiative: a narrative review of psychological and social issues in back pain in low- and middle-income communities. Eur Spine J. https ://doi.org/10.1007/s00586-017-5434-7

31. Chou R, Cote P, Randhawa K, Torres P, Yu H, Nordin M et al (2018) The Global Spine Care Initiative: applying evidencebased guidelines on the non-invasive management of back and neck pain to low- and middle-income communities. Eur Spine J. https://doi.org/10.1007/s00586-017-5433-8

32. Green BN, Johnson CD, Haldeman S, Kane EJ, Clay MB, Griffith E et al The Global Spine Care Initiative: public health and prevention interventions for common spine disorders in low- and middle-income communities. Eur Spine J. https://doi. org/10.1007/s00586-018-5635-8

33. Haldeman S, Johnson CD, Chou R, Nordin M, Côté P, Hurwitz EL et al The Global Spine Care Initiative: classification system for spine-related concerns. Eur Spine J. https://doi.org/10.1007/ s00586-018-5724-8

34. Haldeman S, Johnson CD, Chou R, Nordin M, Côté P, Hurwitz EL et al. The Global Spine Care Initiative: care pathway for people with spine-related concerns. Eur Spine J. https://doi. org/10.1007/s00586-018-5721-y

35. Haldeman S, Nordin M, Chou R, Côté P, Hurwitz EL, Johnson $\mathrm{CD}$, Randhawa $\mathrm{K}$ et al The Global Spine Care Initiative: World Spine Care executive summary on reducing spine-related disability in low- and middle-income communities. Eur Spine J. https://doi.org/10.1007/s00586-018-5722-x

36. Kopansky-Giles D, Johnson CD, Haldeman S, Chou R, Côté $\mathrm{P}$, Green BN, Nordin $\mathrm{M}$ et al The Global Spine Care Initiative: resources to implement a spine care program. Eur Spine J. https ://doi.org/10.1007/s00586-018-5725-7

37. Green BN, Johnson CD, Haldeman S, Griffith E, Clay MB, Kane EJ et al (2018) A scoping review of biopsychosocial risk factors and co-morbidities for common spinal disorders. PLoS ONE 13(6):e0197987

38. Nordin M, Randhawa K, Torres P, Yu H, Haldeman S, Brady O et al (2018) The Global Spine Care Initiative: a systematic review for the assessment of spine-related complaints in populations with limited resources and in low- and middle-income communities. Eur Spine J. https://doi.org/10.1007/s00586-017-5446-3

39. Speerin R, Slater H, Li L, Moore K, Chan M, Dreinhöfer K et al (2014) Moving from evidence to practice: models of care for the prevention and management of musculoskeletal conditions. Best Pract Res Clin Rheumatol 28(3):479-515

40. Davidson P, Halcomb E, Hickman L, Phillips J, Graham B (2006) Beyond the rhetoric: What do we mean by a 'model of care'? Aust J Adv Nurs 23(3):47-55

41. Stolp KA (2011) The rehabilitation model of care: when old becomes new. PM \& R 3(4):289-292

42. Bernabeo E, Holmboe ES (2013) Patients, providers, and systems need to acquire a specific set of competencies to achieve truly patient-centered care. Health Aff 32(2):250-258

43. Mead N, Bower P (2000) Patient-centredness: a conceptual framework and review of the empirical literature. Soc Sci Med 51(7):1087-1110

44. Chou L, Ranger TA, Peiris W, Cicuttini FM, Urquhart DM, Sullivan K et al (2018) Patients' perceived needs of healthcare providers for low back pain management: a systematic scoping review. Spine J 18:691-711

45. Otero C, Luna D, Marcelo A, Househ M, Mandirola H, Curioso W et al (2015) Why patient centered care coordination is important in developing countries? Yearb Med Inform 24(01):30-33

46. Chaple M, Sacks S, Randell J, Kang B (2016) A technical assistance framework to facilitate the delivery of integrated behavioral health services in federally qualified health centers (FQHCs). J Subst Abuse Treat 60:62-69

47. Elder CR, DeBar LL, Ritenbaugh C, Rumptz MH, Patterson C, Bonifay A et al (2017) Health care systems support to enhance patient-centered care: lessons from a primary carebased chronic pain management initiative. Perm J 21:16-101

48. Jacobs JJ, King TR, Klippel JH, Berven SH, Burr DB, Caskey PM et al (2013) Beyond the decade: strategic priorities to 
reduce the burden of musculoskeletal disease. J Bone Jt Surg Am 95(17):e1251-e1256

49. De Man Jerone M, MAc NS, HPPF EWBM, Leyse M, Josefien Van Olmen M, Criel B (2016) Patient-centered care and people-centered health systems in sub-Saharan Africa: Why so little of something so badly needed? Int J 6(3):162-173

50. Jongen CS, McCalman J, Bainbridge RG (2017) The implementation and evaluation of health promotion services and programs to improve cultural competency: a systematic scoping review. Front Publ Health 5:24

51. Organization WH (2016) Framework on integrated, peoplecentred health services. Report by the Secretariat. WHO, Geneva Retrieved from www.whoint/servicedeliverysafety/ areas/people-centred-care/en/on 18

52. Maher C, Underwood M, Buchbinder R (2017) Non-specific low back pain. The Lancet 389(10070):736-747

53. Di Iorio A, Abate M, Guralnik JM, Bandinelli S, Cecchi F, Cherubini A et al (2007) From chronic low back pain to disability, a multifactorial mediated pathway: the InCHIANTI study. Spine 32(26):E809

54. Johnson C, Green BN (2009) Public health, wellness, prevention, and health promotion: considering the role of chiropractic and determinants of health. J Manip Physiol Ther 32(6):405-412

55. White F (2015) Primary health care and public health: foundations of universal health systems. Med Princ Pract 24(2):103-116

56. Waddell G (1987) Volvo award in clinical sciences. A new clinical model for the treatment of low-back pain. Spine (Phila Pa 1976) 12(7):632-644

57. Toro N (2015) Who global strategy on integrated people-centred health services (IPCHS)/Estrategia mundial en servicios de salud integrada centrado en las personas (IPCHS). Int J Integr Care $15(8)$

58. Organization WH (2009) Improving health systems and services for mental health

59. Isaacs D, Fitzgerald D (1999) Seven alternatives to evidence based medicine. BMJ 319(7225): 1618

60. Sackett DL, Rosenberg WM, Gray JA, Haynes RB, Richardson WS (2007) Evidence based medicine: what it is and what it isn't. 1996. Clin Orthop Relat Res 455:3-5

61. Sackett D, Richardson W, Rosenberg W, Haynes R (2000) Evidence-based medicine: how to practice and teach EBM, 2nd edn. Churchill-Livingstone, London

62. Corrigan JM (2005) Crossing the quality chasm. Building a better delivery system. National Academy of Engineering and Institute of Medicine. 2005. Building a Better Delivery System: A New Engineering/Health Care Partnership. Washington, DC: The National Academies Press. https://doi.org/10.17226/11378

63. Boon H, Verhoef M, O'Hara D, Findlay B (2004) From parallel practice to integrative health care: a conceptual framework. BMC Health Serv Res 4(1):15

64. Lemieux-Charles L, McGuire WL (2006) What do we know about health care team effectiveness? A review of the literature. Med Care Res Rev 63(3):263-300

65. World Health Organization (2010) Framework for action on interprofessional education and collaborative practice. Geneva: World Health Organization. http://www.who.int/hrh/resources/ framework_action/en/

66. Organization WH (2015) Integrated health services-what and why? Technical brief no1, May 2008, Geneva: WHO 2008. transition. Lancet 386(10009):2145-2191

67. Valentijn PP, Schepman SM, Opheij W, Bruijnzeels MA (2013) Understanding integrated care: a comprehensive conceptual framework based on the integrative functions of primary care. Int J Integr Care 13 Jan-Mar, URN:NBN:NL:UI:10-1-114415
68. Gröne O, Garcia-Barbero M (2001) Integrated care. Int J Integr Care 1(2):e21

69. Suter E, Oelke ND, Adair CE, Armitage GD (2009) Ten key principles for successful health systems integration. Healthc $Q$ (Toronto, Ont) 13:16

70. Pruitt S, Epping-Jordan J (2002) Innovative care for chronic conditions: building blocks for action: global report: World Health Organization

71. Hwang W, Chang J, LaClair M, Paz H (2013) Effects of integrated delivery system on cost and quality. Am J Managed Care 19(5):e175-e184

72. Shiri R, Coggon D, Falah-Hassani K (2017) Exercise for the prevention of low back pain: systematic review and meta-analysis of controlled trials. Am J Epidemiol 187(5):1093-1101

73. Organization WH (2015) WHO global strategy on people-centred and integrated health services: interim report

74. Carey TS, Garrett J, Jackman A, McLaughlin C, Fryer J, Smucker DR (1995) The outcomes and costs of care for acute low back pain among patients seen by primary care practitioners, chiropractors, and orthopedic surgeons. The North Carolina Back Pain Project. N Engl J Med 333(14):913-917

75. Traeger AC, Hubscher M, Henschke N, Moseley GL, Lee H, McAuley JH (2015) Effect of primary care-based education on reassurance in patients with acute low back pain: systematic review and meta-analysis. JAMA Intern Med 175(5):733-743

76. Fritz JM, Kim M, Magel JS, Asche CV (2017) Cost-effectiveness of primary care management with or without early physical therapy for acute low back pain: economic evaluation of a randomized clinical trial. Spine 42(5):285-290

77. Frogner BK, Harwood K, Andrilla CHA, Schwartz M, Pines JM (2018) Physical therapy as the first point of care to treat low back pain: an instrumental variables approach to estimate impact on opioid prescription, health care utilization, and costs. Health Serv Res. https://doi.org/10.1111/1475-6773.12984

78. Childs JD, Fritz JM, Wu SS, Flynn TW, Wainner RS, Robertson EK et al (2015) Implications of early and guideline adherent physical therapy for low back pain on utilization and costs. BMC Health Serv Res 15:150

79. Kwon B, Tromanhauser SG, Banco RJ (2007) The spine service line: optimizing patient-centered spine care. Spine 32(11):S44-S48

80. Almeida M, Saragiotto B, Richards B, Maher CG (2018) Primary care management of non-specific low back pain: key messages from recent clinical guidelines. Med J Aust 208(6):272-275

81. Bardin LD, King P, Maher CG (2017) Diagnostic triage for low back pain: a practical approach for primary care. Med J Aust 206:268-273

82. Oliveira CB, Maher CG, Pinto RZ, Traeger AC, Lin C-WC, Chenot J-F et al (2018) Clinical practice guidelines for the management of non-specific low back pain in primary care: an updated overview. Eur Spine J 1-13. https://doi.org/10.1007/s0058 6-018-5673-2

83. Lisi AJ, Salsbury SA, Hawk C, Vining RD, Wallace RB, Branson $R$ et al (2018) chiropractic integrated care pathway for low back pain in veterans: results of a Delphi consensus process. J Manip Physiol Ther 41(2):137-148

84. Goertz CM, Long CR, Vining RD, Pohlman KA, Walter J, Coulter I (2018) Effect of usual medical care plus chiropractic care versus usual medical care alone on pain and disability among US service members with low back pain: a comparative effectiveness clinical trial. JAMA Netw Open 1(1):e180105-e

85. Chou R, Deyo R, Friedly J, Skelly A, Hashimoto R, Weimer M et al (2017) Nonpharmacologic therapies for low back pain: a systematic review for an American College of Physicians clinical practice guideline. Ann Intern Med 166(7):493-505 
86. Cherkin DC, Sherman KJ, Balderson BH, Cook AJ, Anderson ML, Hawkes RJ et al (2016) Effect of mindfulness-based stress reduction versus cognitive behavioral therapy or usual care on back pain and functional limitations in adults with chronic low back pain: a randomized clinical trial. JAMA 315(12):1240-1249

87. Pinto AD, Bloch G (2017) Framework for building primary care capacity to address the social determinants of health. Can Fam Phys 63(11):e476-e482

88. Boakye O, Birney A, Suter E, Phillips LA, Suen VY (2016) Scope of practice review: providers for triage and assessment of spine-related disorders. J Multidiscip Healthc 9:227

89. Bitton A, Ratcliffe HL, Veillard JH, Kress DH, Barkley S, Kimball M et al (2017) Primary health care as a foundation for strengthening health systems in low-and middle-income countries. J Gen Intern Med 32(5):566-571

90. Stanos S, Brodsky M, Argoff C, Clauw DJ, D’Arcy Y, Donevan $S$ et al (2016) Rethinking chronic pain in a primary care setting. Postgrad Med 128(5):502-515

91. Perry HB, Zulliger R, Rogers MM (2014) Community health workers in low-, middle-, and high-income countries: an overview of their history, recent evolution, and current effectiveness. Annu Rev Publ Health 35:399-421

92. Panel IECE (2016) Core competencies for interprofessional collaborative practice: 2016 update. Interprofessional Education Collaborative, Washington, DC

93. Organization WH (2015) World report on ageing and health: World Health Organization

94. Grol R, Grimshaw J (2003) From best evidence to best practice: effective implementation of change in patients' care. The Lancet 362(9391): 1225-1230

95. Granger CB, Xavier D (2018) Improving evidence for implementation of guideline-based care in low- and middle-income countries. JAMA 319(6):554-556

96. Kadu MK, Stolee P (2015) Facilitators and barriers of implementing the chronic care model in primary care: a systematic review. BMC Fam Pract 16(1):12

97. Mills A (2014) Health care systems in low-and middle-income countries. N Engl J Med 370(6):552-557

98. Briggs AM, Jordan J, Jennings M, Speerin R, Chua J, Bragge $\mathrm{P}$, Slater H A framework to evaluate musculoskeletal models of care. https://nzoa.org.nz/system/files/Framework-to-EvaluateMusculoskeletal-MoC.pdf

99. Butterfoss FD (2007) Coalitions and partnerships in community health. Wiley, Hoboken
100. Fertman CI, Allensworth DD (2016) Health promotion programs: from theory to practice. Wiley, Hoboken

101. Party SCW (2009) Spinal pain model of care. Perth: HealthNetworks Branch, Department of Health, Western Australia

102. Bryant-Lukosius D, Spichiger E, Martin J, Stoll H, Kellerhals SD, Fliedner M et al (2016) Framework for evaluating the impact of advanced practice nursing roles. J Nurs Scholarsh 48(2):201-209

103. French SD, Green SE, O'Connor DA, McKenzie JE, Francis JJ, Michie S et al (2012) Developing theory-informed behaviour change interventions to implement evidence into practice: a systematic approach using the Theoretical Domains Framework. Implement Sci 7(1):38

104. Veillard J, Cowling K, Bitton A, Ratcliffe H, Kimball M, Barkley $S$ et al (2017) Better measurement for performance improvement in low-and middle-income countries: the primary health care performance initiative (PHCPI) experience of conceptual framework development and indicator selection. Milbank Q 95(4):836-883

105. Al Zoubi FM, Eilayyan O, Mayo NE, Bussières AE (2017) Evaluation of cross-cultural adaptation and measurement properties of STarT back screening tool: a systematic review. J Manip Physiol Ther 40(8):558-572

106. Eaton J, McCay L, Semrau M, Chatterjee S, Baingana F, Araya R et al (2011) Scale up of services for mental health in low-income and middle-income countries. The Lancet 378(9802):1592-1603

107. Gaziano TA, Pagidipati N (2013) Scaling up chronic disease prevention interventions in lower- and middle-income countries. Annu Rev Publ Health 34:317-335

108. Organization WH. Scaling up health services: challenges and choices 2008. http://www.who.int/healthsystems/topics/delivery/ technical_brief_scale-up_june12.pdf

109. Foster NE, Anema JR, Cherkin D, Chou R, Cohen SP, Gross DP et al (2018) Prevention and treatment of low back pain: evidence, challenges, and promising directions. The Lancet 391(10137):2368-2383

110. WHO (2015) WHO global strategy on integrated people-centred health services 2016-2026: placing people and communities at the centre of health services

111. Van Lerberghe W (2008) The world health report 2008: primary health care: now more than ever. World Health Organization

112. Nations U (2015) Transforming our world: the 2030 agenda for sustainable development. Resolution adopted by the General Assembly

113. Grumbach K, Bodenheimer T (1995) The organization of health care. JAMA 273(2):160-167

\section{Affiliations}

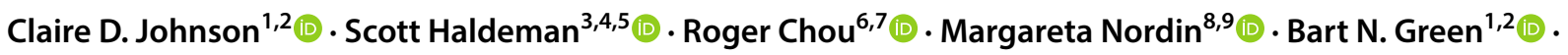
Pierre Côté ${ }^{10,11}$ (I) Eric L. Hurwitz ${ }^{12}$ (D) Deborah Kopansky-Giles ${ }^{13,14}$ (D) Emre Acaroğlu ${ }^{15}$ (D) Christine Cedraschi ${ }^{16,17}$ (1) Arthur Ameis ${ }^{18}$ (1) Kristi Randhawa $^{10,11}$ (D) Ellen Aartun ${ }^{11} \cdot$ Afua Adjei-Kwayisi $^{19}{ }^{(1)}$. Selim Ayhan ${ }^{20}$ - Amer Aziz ${ }^{21}$. Teresa Bas ${ }^{22}$. Fiona Blyth ${ }^{23}$. David Borenstein ${ }^{24}$ - O'Dane Brady ${ }^{25}$ (D) Peter Brooks ${ }^{26}$. Connie Camilleri ${ }^{27}$. Juan M. Castellote ${ }^{28}$. Michael B. Clay ${ }^{29}$. Fereydoun Davatchi ${ }^{30}$ (D) Jean Dudler ${ }^{31}$ - Robert Dunn ${ }^{32}$ - Stefan Eberspaecher ${ }^{33}$. Juan Emmerich ${ }^{34}$. Jean Pierre Farcy ${ }^{35}$. Norman Fisher-Jeffes ${ }^{36} \cdot$ Christine Goertz $^{37}$ (1) Michael Grevitt ${ }^{38} \cdot$ Erin A. Griffith $^{39} \cdot$ Najia Hajjaj-Hassouni $^{40}{ }^{(1)}$. Jan Hartvigsen ${ }^{41,42}$ (D) . Maria Hondras ${ }^{43}$ (i) - Edward J. Kane ${ }^{44}$ (i) . Julie Laplante ${ }^{45}$. Nadège Lemeunier ${ }^{46}$. John Mayer ${ }^{47} \cdot$ Silvano Mior ${ }^{48}$ - Tiro Mmopelwa ${ }^{49} \cdot$ Michael Modic $^{50} \cdot$ Jean Moss $^{48} \cdot$ Rajani Mullerpatan $^{51}$. Elijah Muteti ${ }^{52} \cdot$ Lillian Mwaniki $^{53} \cdot$ Madeleine Ngandeu-Singwe $^{54} \cdot$ Geoff Outerbridge $^{55}$ (1) Shanmuganathan Rajasekaran ${ }^{56} \cdot$ Heather Shearer $^{11} \cdot$ Matthew Smuck $^{57} \cdot$ Erkin Sönmez $^{58}$ (1) Patricia Tavares $^{48}$. Anne Taylor-Vaisey ${ }^{11}$. Carlos Torres $^{59} \cdot$ Paola Torres $^{60} \cdot$ Alexander van der Horst $^{61,62}$. Leslie Verville $^{10,11}$. Emiliano Vialle ${ }^{63}$. Gomatam Vijay Kumar ${ }^{64}$. Adriaan Vlok ${ }^{65}$. William Watters III ${ }^{66,67}$. Chung Chek Wong ${ }^{68}$. Jessica J. Wong ${ }^{11} \cdot$ Hainan Yu ${ }^{10,11}$ (D) Selcen Yüksel ${ }^{69}$ (]) 
1 National University of Health Sciences, Lombard, IL, USA

2 Qualcomm Health Center, Stanford Health Care, San Diego, CA, USA

3 Department of Epidemiology, School of Public Health, University of California Los Angeles, Los Angeles, CA, USA

4 Department of Neurology, University of California, Irvine, Irvine, CA, USA

5 World Spine Care, Santa Ana, CA, USA

6 Department of Medical Informatics and Clinical Epidemiology, Oregon Health and Science University, Portland, OR, USA

7 Department of Medicine, Oregon Health and Science University, Portland, OR, USA

8 Departments of Orthopedic Surgery and Environmental Medicine, New York University, New York, NY, USA

9 World Spine Care Europe, Holmfirth, UK

10 Faculty of Health Sciences, University of Ontario Institute of Technology, Oshawa, Canada

11 UOIT-CMCC Centre for Disability Prevention and Rehabilitation, Toronto, Canada

12 Office of Public Health Studies, University of Hawai i, Mānoa, Honolulu, HI, USA

13 Department of Research, Canadian Memorial Chiropractic College, Toronto, ON, Canada

14 Department of Family and Community Medicine, University of Toronto, Toronto, ON, Canada

15 ARTES Spine Center, Ankara, Turkey

16 Division of General Medical Rehabilitation, Multidisciplinary Pain Centre, Geneva University Hospitals, Geneva, Switzerland

17 Division of Clinical Pharmacology and Toxicology, Multidisciplinary Pain Centre, Geneva University Hospitals, Geneva, Switzerland

18 Certification Program in Insurance Medicine and MedicoLegal Expertise, Faculty of Medicine, University of Montreal, Toronto, ON, Canada

19 Ridge Regional Hospital, Ghana World Spine Care, Accra, Greater Accra, Ghana

20 ARTES Spine Center, Acibadem University, Ankara, Turkey

21 Orthopaedics and Spine Department, Ghurki Trust Teaching Hospital, Lahore, Punjab, Pakistan

22 Department of Surgery, Hospital Universitario and Politecnico La Fe, Valencia University, Valencia, Spain

23 Concord Clinical School, University of Sydney, Concord, NSW, Australia

24 Arthritis and Rheumatism Associates, The George Washington University Medical Center, Potomac, MD, USA

25 World Spine Care, Tampa, FL, USA

26 Centre for Health Policy, School of Population and Global Health, University of Melbourne, Toorak, Melbourne, VIC, Australia

27 St Michael's Hospital, North York, ON, Canada
28 National School of Occupational Medicine, Carlos III Institute of Health and Physical Medicine and Rehabilitation, University of Complutense, Madrid, Madrid, Spain

29 Rehabilitation Care Line, Physical Medicine and Rehabilitation, Cincinnati Veterans Affairs Medical Center, Cincinnati, OH, USA

30 Rheumatology Research Center, Tehran University of Medical Sciences, Tehran, Tehran, Iran

31 Department of Rheumatology, HFR Fribourg - Hospital Cantonal, Fribourg, FR, Switzerland

32 Department of Orthopaedic Surgery, Groote Schuur Hospital, University of Cape Town, Cape Town, Western Cape, South Africa

33 Department of Orthopedics, Princess Marina Hospital, Gaborone, Botswana

34 University of La Plata, La Plata, Buenos Aires, Argentina

35 Department of Orthopedic Surgery (retired), New York University, Piermont, NY, USA

36 Private Practice - Panorama Medi Clinic and Sessional Consultant, Groote Schuur Hospital, Cape Town, Western Cape, South Africa

37 Palmer College of Chiropractic, The Spine Institute for Quality, Davenport, IA, USA

38 Nottingham University Hospitals, Edwalton, Nottingham, UK

39 Emergency Medicine, Carlsbad, CA, USA

40 Mohammed VI University of Health Sciences (UM6SS), Casablanca, Morocco

41 Department of Sports Science and Clinical Biomechanics, University of Southern Denmark, Odense, Denmark

42 Nordic Institute of Chiropractic and Clinical Biomechanics, Odense, Denmark

43 Department of Anesthesiology, University of Kansas Medical Center, Kansas City, KS, USA

44 College of Rehabilitative Sciences, Doctor of Physical Therapy Program, University of St. Augustine for Health Sciences, San Marcos, CA, USA

45 School of Sociological and Anthropological Studies, University of Ottawa, Ottawa, ON, Canada

46 Institut Franco-Européen de Chiropraxie, Toulouse, France

47 U.S. Spine and Sport Foundation, San Diego, CA, USA

48 Canadian Memorial Chiropractic College, Toronto, ON, Canada

49 ARTES Ankara Spine Centre, Life Gaborone Hospital, Gaborone, Botswana

50 Cleveland Clinic, Lerner College of Medicine, Cleveland, $\mathrm{OH}$, USA

51 MGM School of Physiotherapy, Mahatma Gandhi Mission Institute of Health Sciences, Navi Mumbai, Maharashtra, India

52 Moi Teaching and Referral Hospital, Moi University, Eldoret, Kenya

53 Law Society of Kenya, Nairobi, Kenya 
54 Faculty of Medicine and Biomedical Sciences, The University of Yaoundé I, Yaoundé, Center Region, Cameroon

55 World Spine Care, Canadian Memorial Chiropractic College, Chelsea, QC, Canada

56 Department of Orthopaedics and Spine Surgery, Ganga Hospital, Coimbatore, Tamilnadu, India

57 Department of Orthopaedic Surgery, Stanford University, Redwood City, CA, USA

58 Department of Neurological Surgery, Başkent University School of Medicine, Ankara, Turkey

59 Department of Medical Imaging, The Ottawa Hospital, University of Ottawa, Ottawa, ON, Canada

60 Exercise Science Laboratory, Faculty of Medicine, School of Kinesiology, Universidad Finis Terrae, Santiago, Chile

61 Department of Surgery, University of Namibia, Windhoek, Namibia
62 Central Hospital, Windhoek, Namibia

63 Catholic University of Parana, Curitiba, Paraná, Brazil

64 AMRI Hospitals, Salt Lake, Kolkata, West Bengal, India

65

Division of Neurosurgery, University of Stellenbosch, Bellville, Western Cape, South Africa

66 Department of Orthopedic, Weill Cornell Medical School, Institute of Academic Medicine, Houston Methodist Hospital, Houston, TX, USA

67 Department of Orthopedic Surgery, University of Texas Medical Branch, Galveston, TX, USA

68 Sarawak General Hospital, Kuching, Sarawak, Malaysia

69 Department of Biostatistics, Ankara Yildirim Beyazit University Medical School, Ankara, Turkey 